INRA Prod. Anim., 1994, 7 (1), 29 - 39.

\section{A. POINTILLART}

INRA Laboratoire de Nutrition et Sécurité Alimentaire

78352 Jouy-en-Josas Cedex

\section{Phytates, phytases : leur importance dans l'alimentation des monogastriques}

Le phosphore végétal des grains et issues, pour l'essentiel sous forme de phytates, est diversement utilisé par les volailles et les porcs. La phytase de certaines céréales ou l'addition de phytases microbiennes influe considérablement sur cette utilisation. La présence de ces enzymes peut donc permettre de substantielles économies dans l'addition de phosphates minéraux et réduire considérablement l'excrétion de phosphore dans les effluents d'élevage. Un peu partout en Europe et aux Etats-Unis, l'idée (et la pratique) d'ajouter ces phytases se répand, il nous a donc paru nécessaire de faire le point sur ce sujet.

L'augmentation des rejets de phosphore dans les zones à forte concentration d'élevages de monogastriques, les soucis croissants vis-à-vis de ce type de pollution (taxée aux Pays-Bas), la mise au point de phytases d'origine microbienne favorisant la digestion du phosphore végétal, dont les aliments des porcs, des volailles (voire de l'homme) sont riches, ont focalisé ces dernières années

\section{Résumé}

Le phosphore des végétaux constitue la majeure partie (2/3 à 3/4) des apports de phosphore chez les monogastriques. Il est pour l'essentiel $(2 / 3$ à $3 / 4)$ sous forme d'acide phytique ou de phytates (de $\mathrm{Na}, \mathrm{K}$, ...). L'utilisation digestive de cette forme de $\mathrm{P}$ par l'animal dépend pour beaucoup de l'addition ou de la présence naturelle de phytase et varie de moins de $20 \%$ pour les régimes dépourvus à plus de $60 \%$ pour ceux enrichis en phytase microbienne ( $\geq 1000$ unités d'enzyme/kg d'aliment). Cette enzyme, en hydrolysant le phosphore phytique, réputé peu disponible et ayant de nombreuses propriétés antinutritionnelles vis-à-vis de certains cations, permet d'économiser partiellement, voire totalement (porcs), sur les phosphates minéraux ajoutés et de réduire considérablement (jusqu'à 50\%) les teneurs en $P$ des lisiers. Le principal frein à l'utilisation généralisée des phytases, qu'elles soient ajoutées ou naturellement présentes dans certaines céréales comme le blé ou le seigle et leurs sous-produits, reste le problème de leur conservation au cours de la fabrication des aliments : un trop fort échauffement au cours de la granulation $\left(70-80^{\circ} \mathrm{C}\right)$ conduisant à des pertes importantes $\left(30\right.$ à $50 \%$, près de $100 \%$ au-delà de $\left.80^{\circ} \mathrm{C}\right)$ de l'activité phytasique. Enfin, l'étude systématique des correspondances entre activité phytasique des céréales et digestibilités de $P$ de celles-ci reste à faire. l'attention sur le phosphore phytique et son utilisation, notamment chez le porc et les volailles. Des travaux datant de plus de 20 ans aux USA (Nelson et al 1971) et en France à l'INRA (Guéguen et al 1968), qui avaient été plus ou moins abandonnés, ont été réactualisés et poursuivis à l'INRA (Laboratoire de Nutrition et Sécurité Alimentaire, Jouy-enJosas et Station de Recherches Avicoles, Nouzilly) et surtout en Hollande (Jongbloed, IVVO). Dans les années 80, les travaux de l'INRA abordaient le problème de la disponibilité variable du phosphore végétal (Sauveur 1983 ), tout en alertant les éleveurs sur d'inutiles excès d'emploi de phosphore dans les aliments, excès de $\mathrm{P}$ minéral souvent très coûteux ; ils avaient donc pour but une meilleure valorisation des apports de $P$ végétal, c'est-à-dire pour l'essentiel (2/3-3/4) de P phytique, valorisation pour l'animal aussi bien qu'économique. A partir de 1987 , les Hollandais, à l'initiative de Jongbloed, ont entrepris une vaste série d'essais, stimulés à la fois par le fait que la concentration d'élevage était devenue telle qu'il fallait trouver des moyens efficaces de réduire les effluents de $P$ (et d'azote) et par la mise au point, par GistBrocades, d'une phytase d'origine fongique (Aspergillus niger/ficuum) se révélant assez rapidement efficiente. Ainsi cette enzyme, en augmentant considérablement la digestibilité de $\mathrm{P}$ végétal, permettait de réduire fortement 
les apports de $\mathrm{P}$ nécessaires aux volailles et aux porcins pour assurer de bonnes performances. Les dernières revues générales (Sauveur 1989 et 1993) étant avant tout consacrées aux volailles, la présente revue sera davantage orientée vers l'espèce porcine. En effet, depuis quelques années, la formulation alimentaire chez les volailles utilise le "phosphore disponible" et non plus le P total qui sert encore de base aux recommandations INRA pour le porc tandis que les formulateurs raisonnent eux aussi de plus en plus en $\mathrm{P}$ disponible.

\section{1 / Acide phytique et phytates}

\section{1 / Aspects physico-chimiques}

L'acide phytique ou acide myo-inositol hexaphosphorique (IP6) est composé d'un radical inositol estérifié par 6 radicaux phosphate euxmêmes impliqués dans des liaisons avec les cations. C'est un composant essentiel des graines (céréales et légumineuses), généralement classé avec les fibres pour les implications nutritionnelles. Toutefois, il est clairement démontré que ce sont les phytates, plus que les fibres (celluloses, hémicelluloses, pectines, etc.) qui sont responsables des problèmes de disponibilité des minéraux, notamment $\mathrm{Ca}, \mathrm{Mg}, \mathrm{Zn}, \mathrm{Fe}, \mathrm{Cu}$ (revue de Pointillart et Guéguen 1992). Chez le porc, la maladie du son (ostéofibrose avec atrophie du groin) ainsi que la parakératose (carence en zinc pouvant être liée à une consommation excessive d'issues de céréales) (Oberleas et al 1962) constituent des exemples historiques des effets d'un excès de $P$ phytique alimentaire sur l'indisponibilité des minéraux d'intérêt nutritionnel.

La molécule présente une teneur élevée en $P(28,2 \%)$ et ses 6 radicaux acide phosphorique ont une affinité variable pour les divers cations. La stabilité des phytates et leur affinité pour les cations varient ainsi : $\mathrm{Fe}<\mathrm{Ca}<\mathrm{Mn}<\mathrm{Co}<\mathrm{Cu}<\mathrm{Zn}$ (Erdman 1979) avec des variantes pour l'ordre $\mathrm{Fe} / \mathrm{Ca}$ et $\mathrm{Cu} / \mathrm{Zn}$. Les phytates naturels sont plutôt des phytates de
$\mathrm{Mg}+\mathrm{K}$, solubles mais déplacés par les autres cations présents dans le régime, en particulier $\mathrm{Ca}, \mathrm{Zn}$ et $\mathrm{Fe}$. Une molécule d'acide phytique capte en moyenne 3 à 6 moles de $\mathrm{Ca}$ pour former des phytates insolubles au pH intestinal, ce qui rend indisponible et le phosphore et le calcium.

L'acide phytique représente à la fois une réserve de phosphore et de glucide qui seront utilisés par la plantule lors de la germination. Dans ce cas, l'acide phytique ou les phytates de $\mathrm{Na}, \mathrm{K}, \mathrm{Mg}$... doivent être hydrolysés pour libérer les orthophosphates et l'inositol, l'enzyme (ou les enzymes suivant les espèces végétales) responsable étant une phosphatase particulière, acide, la phytase (EC 3138)

La déphosphorylation de l'acide phytique (IP6) aboutit à de l'inositol-5 phosphates (IP5),-4 phosphates (IP4),-3 (IP3),-2, (IP2),1 phosphate (IP1), les 3 derniers produits étant susceptibles de passer la barrière intestinale (l'inositol-3-phosphates, IP3, est un médiateur biochimique cellulaire connu). Dans les graines, la quasi totalité de l'acide phytique est constituée d'inositol-6 phosphates mais la trituration, le broyage, l'humidification, peuvent amorcer l'hydrolyse, au moins chez les graines à forte activité phytasique, et parfois des proportions significatives d'inositol-phosphates, supposés absorbables, peuvent apparaître (tableau 1) (Bos 1986).

Le dosage des phytates consistait jusqu'ici à précipiter le phosphore phytique sous forme de phytates de fer (à l'aide de $\mathrm{FeCl}_{3}$ ) et de doser $\mathrm{P}$ après minéralisation de ce précipité. Maintenant, le dosage par chromatographie liquide à haute pression (CLHP ou HPLC en anglais) permet la séparation des principaux inositol-phosphates.

\section{2 / Localisation des phytates (tableau 2)}

Dans les céréales, l'acide phytique est associé à des structures particulières du grain. Dans le riz et le blé, il est présent dans le germe mais avant tout dans les enveloppes (péricarpe, testa et aleurone), les principaux

Tableau 1. Répartition des différents inositol-phosphates dans quelques matières premières.

\begin{tabular}{|l|c|c|c|c|}
\hline & $\begin{array}{c}(\mathbf{1}) \\
\begin{array}{c}\text { Phosphore } \\
\text { phytique } \\
\text { total }\end{array}\end{array}$ & $\begin{array}{c}\text { IP) } \\
\text { IP6 }\end{array}$ & $\begin{array}{c}\text { (3) } \\
\text { IP4 } \\
+ \\
\text { IP5 }\end{array}$ & $\begin{array}{c}\text { (4) } \\
\text { Autres IP } \\
\text { en \% P } \\
\text { phytique total }\end{array}$ \\
\hline Blé & 3,1 & 2,3 & 0,3 & 16 \\
Remoulage de blé & 9,7 & 8,9 & 1,1 & - \\
Maïs & 2,4 & 2,1 & 0,3 & - \\
Pois & 2,6 & 1,9 & 0,3 & - \\
Maïs gluten-feed & 5,9 & 4,9 & 1,0 & 7 \\
Soja broyé & 4,2 & 3,6 & 0,3 & \\
\hline
\end{tabular}

(1) : phosphore phytique total du produit rapporté à sa matière sèche en $\mathrm{g} / \mathrm{kg}$

(2) : idem pour l'inositol-6 phosphates

(3): idem pour la somme inositol-4 phosphates et -5 phosphates

(4) : proportion d'inositol-phosphates (\% phosphore phytique total) qui ne sont ni IP6, ni IP5, ni IP4 et donc a priori plus absorbables. Calculs d'après Bos (1986). 
Tableau 2. Localisation de l'acide phytique dans le grain (d'après Reddy et al 1982)

\begin{tabular}{|c|c|c|c|}
\hline Céréale & Echantillon & $\begin{array}{c}\text { P phytique } \% \\
(\mathbf{1})\end{array}$ & $\begin{array}{c}\text { Distribution (2) } \\
\text { en \% du total } \\
\text { dans le grain }\end{array}$ \\
\hline \multirow{2}{*}{ Maïs } & Hybride commercial & 0,25 & - \\
& Endosperme & 0,01 & 3 \\
& Germe & 1,80 & 88 \\
Blé & Cuticule & 0,02 & 0,4 \\
& Tendre & 0,32 & - \\
& Endosperme & traces & 2 \\
& Germe & 1,10 & 13 \\
Riz & Téguments & 0 & 0 \\
& Aleurone & 1,16 & 87 \\
& Brun & 0,25 & - \\
& Endosperme & traces & 1,2 \\
& Germe & 0,98 & 7,6 \\
& Péricarpe & 0,95 & 80 \\
\hline
\end{tabular}

(1) Teneur en phosphore phytique en \% de la partie considérée.

(2) Distribution : la somme supérieure à $100 \%$ s'explique parce que certaines parties analysées en recouvrent partiellement d'autres (blé).

sites d'accumulation étant les grains d'aleurone. Pour le maïs, il est essentiellement dans le germe. Pour les légumineuses (dicotylédones), l'acide phytique est dispersé dans les cotylédons, associé dans les corps protéiques à des inclusions appelées globoïdes.

\section{3 / Abondance du phosphore phytique (tableau 3)}

La nature même des régimes des porcs et des volailles fait que le $\mathrm{P}$ végétal y est abondant, en général de $2 / 3$ à $3 / 4$ de la consommation totale de phosphore. Une grande partie de ce phosphore végétal est sous forme de phytates.

La teneur moyenne en phosphore phytique, aux alentours de $0,2 \%$ de la matière sèche, varie assez peu d'une céréale à l'autre ; les proportions rapportées au phosphore végétal total sont nettement plus variables (de 50 à $85 \%$ ). Cela peut avoir des conséquences non négligeables sur la proportion de $\mathrm{P}$ végétal non phytique (phospholipides, phosphoprotéines, nucléoprotéines...) réputé disponible, c'est-àdire utilisable par l'animal. Pour la plupart des matières premières, $2 / 3$ à $3 / 4$ de leur phosphore total est sous forme phytique à l'exception du pois (environ $50 \%$ ) et du tourteau de soja. Si l'on considère isolément chaque matière première, il n'y a pas de relation directe entre sa teneur en $\mathrm{P}$ total et sa teneur en $P$ phytique, les proportions de l'un par rapport à l'autre variant considérablement d'un échantillon à l'autre (Maupetit et Gatel 1992). Autrement dit, la seule détermination de $P$ total ne permet pas de calculer le $P$ phytique sauf à prendre la fourchette large $2 / 3-3 / 4$. Cependant, ni la teneur en $\mathrm{P}$ total ni la proportion de $\mathrm{P}$ phytique ne suffisent à rendre compte de la digestibilité de $P$ végétal. En effet, pour des teneurs en $\mathrm{P}$ total et phytique identiques, les matières premières ne présentent pas la même digestibilité chez les volailles et les porcs : le phosphore phytique, pour être absorbé par l'animal, doit être dégradé, hydrolysé, par une (ou plusieurs) enzyme, la phytase, présente dans certaines céréales et pas dans d'autres. Il est donc erroné de calculer la partie disponible du phosphore végétal par la formule : $\mathrm{P}$ disponible $=\mathrm{P}$ végétal total $-\mathrm{P}$ phytique, bien que cela ait été une règle d'or largement utilisée jusqu'ici par de nombreux formulateurs.

Tableau 3.Phosphore phytique (en $\mathrm{g} / \mathrm{kg}$ ) des principales matières premières en alimentation animale. Synthèse d'après les données recueillies par Pointillart et Colin (1993), Cromwell (1989), Sauveur (1989), Maupetit et Gatel (1992, ITCF), Eeckhout et De Paepe (1993, communication personnelle). Pour convertir $P$ phytique en acide phytique, diviser par 0,282 .

\begin{tabular}{|l|c|c|}
\hline & P Phytique & $\begin{array}{c}\text { P phytique } \\
\text { /P total (en \%) }\end{array}$ \\
\hline Blé & $1,7-2,5$ & $60-77$ \\
Mais & $1,7-2,2$ & $66-85$ \\
Avoine & $1,9-2,3$ & $55-63$ \\
Orge & $1,9-2,5$ & $51-66$ \\
Triticale & $2,5-2,6$ & $65-68$ \\
Seigle & $2,2-2,5$ & $61-73$ \\
Sorgho & $1,8-2,2$ & $60-74$ \\
Pois* & $1,2-1,7$ & $40-50$ \\
Sons de blé* & $8,1-9,7$ & $70-90$ \\
Remoulages de blé & $4,7-5,8$ & $66-85$ \\
Sons de seigle & 7,6 & 71 \\
Tourteaux & & \\
- Soja & $3,2-3,8$ & $51-61$ \\
- Colza & $6,0-7,3$ & $60-73$ \\
- Tournesol** & $6,2-9,2$ & $73-80$ \\
- Arachide & $3,2-4,3$ & $47-69$ \\
- Coton & $7,0-7,5$ & 70 \\
\hline
\end{tabular}

*Valeurs ITCF non prises en compte :Décortiqué.

\section{Le phosphore phytique représente en moyenne plus des 2/3 du $P$ des matières premières des aliments pour monogastriques.}




\section{2 / Phytases}

Il existe au moins 3 sortes d'activité phytasique : celle de la céréale proprement dite, celle que pourrait présenter le tube digestif de l'animal (de l'intestin ou de sa flore) ou celle qu'on peut rajouter à l'aliment, d'origine microbienne (Aspergillus ficuum ou niger), certaines produites par génie génétique, et dont l'usage se répand aujourd'hui en Europe et aux Etats-Unis, chez les porcs et les volailles.

\section{1 / Phytases végétales}

La phytase végétale, celle du blé notamment, est connue depuis fort longtemps

Figure 1. Activité phytasique des céréales et des tourteaux. Données provenant du LNSA (Pointillart et Colin 1993, non publié), (b) : données LNSA + données de Eeckhout et De Paepe (1993) regroupées. En haut, quelques issues (et le seigle) à très forte activité phytasique (entre 2000 et 8000 unités $/ \mathrm{kg}$ ) et en bas, comparaison entre les céréales à forte activité phytasique et toutes les autres matières premières (échelle différente : de 0 à 1500 unités $/ \mathrm{kg}$ )

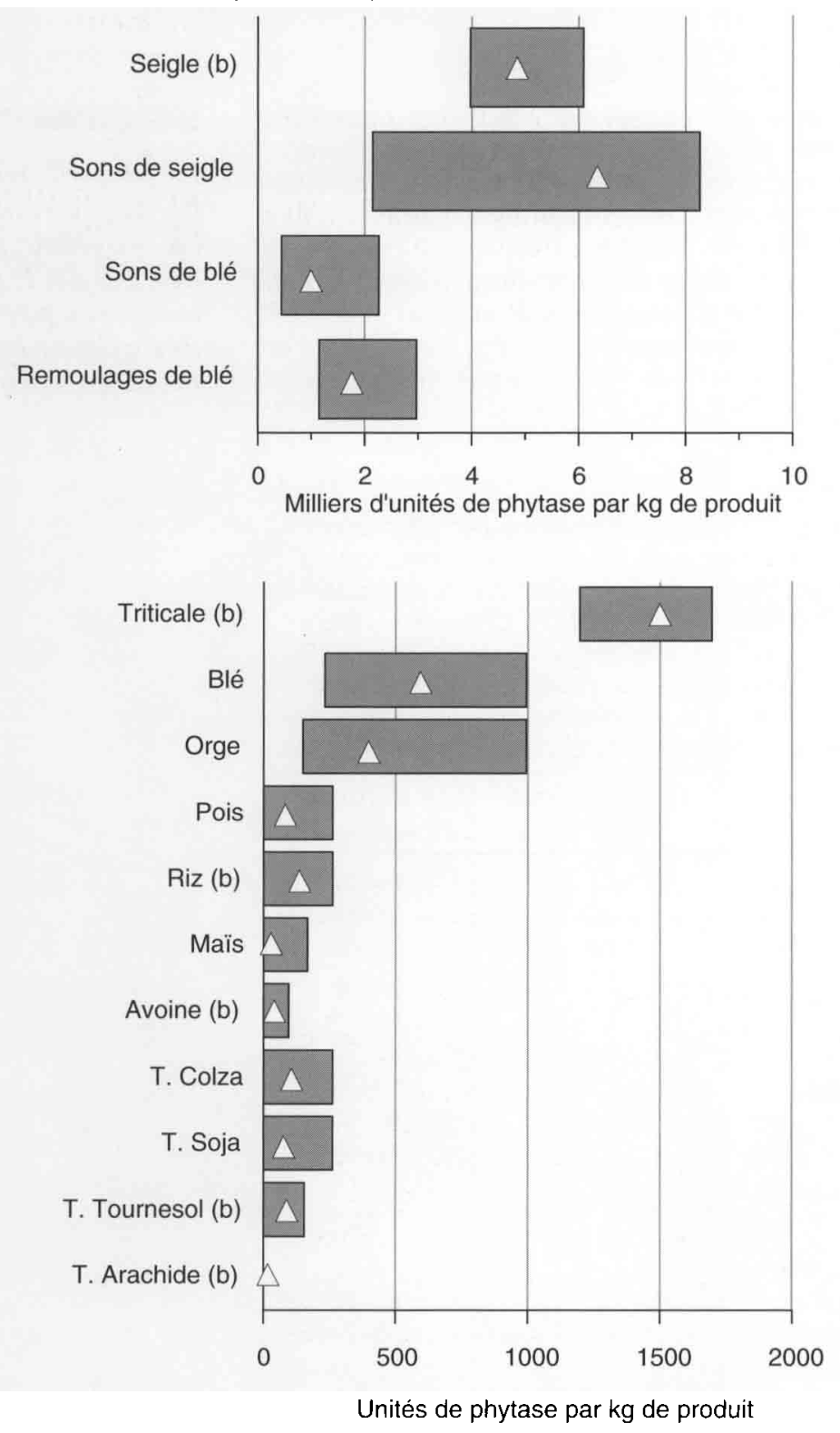

Mini - Maxi

A Valeur moyenne
(Courtois 1947) ; elle a été isolée et caractérisée à partir de sources différentes, blé, maïs, orge, riz, triticale, haricots, courge, etc. (revue de Fourdin 1984).

\section{a / Importance de la phytase}

L'activité phytasique dans les grains et graines varie considérablement suivant l'espèce végétale considérée (tableau 4, figure 1). En fait, assez peu de graines présentent une activité phytasique élevée hormis le blé, le seigle et leur hybride, le triticale (tableau 4). L'activité phytasique de l'orge est extrêmement variable : tantôt l'orge est à classer dans les céréales sans activité, tantôt elle présente une

Tableau 4. Activité phytasique moyenne des matières premières $(U / \mathrm{kg}$, une unité correspond à la libération d'1 $\mu$ mole de $P / m i n$ à partir du substrat phytate de sodium, à $\mathrm{pH} 5,5$ et à $37^{\circ} \mathrm{C}$ ).

\begin{tabular}{|l|c|c|}
\hline Blé & $600 \pm 60$ & $(\mathrm{n}=11)$ \\
Remoulages de blé & $1900 \pm 140$ & $(\mathrm{n}=12)$ \\
Sons de blé & $1100 \pm 120$ & $(\mathrm{n}=24)$ \\
Seigle & $4900 \pm 620$ & $(\mathrm{n}=3)$ \\
Sons de seigle & $6300 \pm 1100$ & $(\mathrm{n}=8)$ \\
Triticale $^{*}$ & $1500 \pm 170$ & $(\mathrm{n}=8)$ \\
Orge & $400 \pm 200$ & $(\mathrm{n}=4)$ \\
Pois & $80 \pm 20$ & $(\mathrm{n}=20)$ \\
Maïs & $30 \pm 15$ & $(\mathrm{n}=7)$ \\
Avoine & $30 \pm 15$ & $(\mathrm{n}=6)$ \\
Riz $^{*}$ & $125 \pm 60$ & $(\mathrm{n}=7)$ \\
Tourteaux & & \\
- Colza & $100 \pm 50$ & $(\mathrm{n}=6)$ \\
- Soja & $60 \pm 30$ & $(\mathrm{n}=8)$ \\
- Tournesol* & $60 \pm 20$ & $(\mathrm{n}=11)$ \\
- Arachide & 0 & $(\mathrm{n}=3)$ \\
\hline
\end{tabular}

Mesures réalisées au LNSA (Pointillart et Colin 1993, données non publiées) *Valeurs LNSA poolées avec celles d'Eeckhout et De Paepe. (1993, communication personnelle). (n) : taille de l'échantillonnage.

Figure 2. Variabilité de l'activité phytasique des pois. Données provenant du LNSA (Colin et Pointillart 1993, non publié).

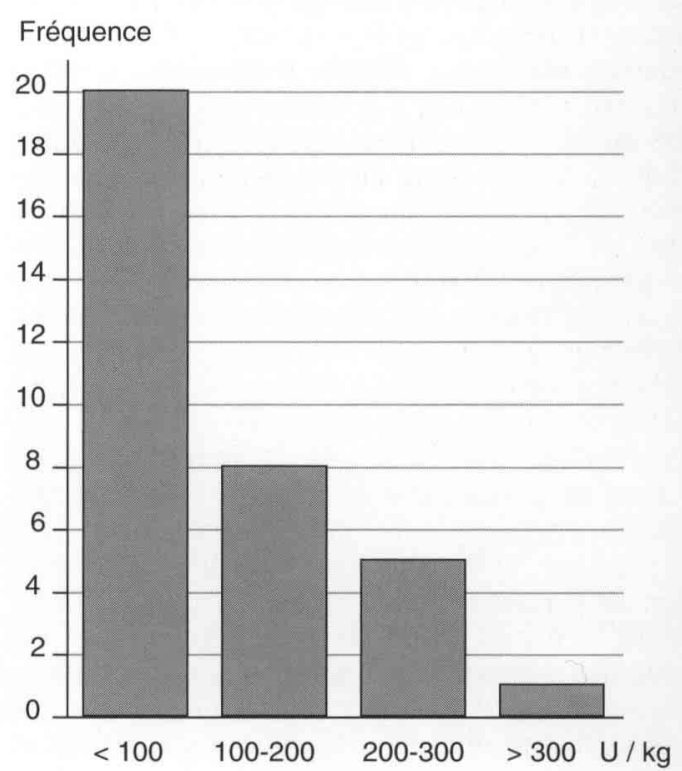


activité phytasique comparable à celle du blé (Latimier et Pointillart 1993), quant au pois, contrairement à ce qui a parfois été écrit, son activité phytasique est le plus souvent négligeable ou nulle (figure 2) ce qui est également le cas des autres matières premières utilisées en alimentation animale, à l'exception des issues de céréales à forte activité phytasique.

Pour les blés et sous-produits du blé, les analyses réalisées à l'INRA indiquaient l'absence de corrélation entre les teneurs en phytates et les activités phytasiques. De même, alors que les teneurs en $P$ total et phytique variaient d'environ $10 \%$, l'activité phytasique variait de 1 à 3 pour les échantillons de blé analysés. Cette très large amplitude de variation se retrouve parfois exagérée, pour l'ensemble des matières premières à haute activité phytasique (figure 1). Cela vient peut être des incertitudes des dosages ou de l'échantillonnage (stockage et taille).

\section{b / Répartition anatomique (tableau 5)}

Pour le blé et le triticale, la phytase est surtout dans les enveloppes (le son) mais elle est plus dispersée dans le grain que ne le sont les phytates. Toutefois, la plus forte activité phytasique correspond également à la plus forte concentration de phytates : dans l'aleurone. Pour simplifier, la phytase des grains et des graines se situe surtout, comme les phytates, dans les enveloppes mais l'endosperme (=amande du grain) présente une forte activité enzymatique en dépit d'une présence négligeable de phytates.

\section{c / Facteurs influençant l'activité phytasique}

- L'humidité : la phytase étant une enzyme hydrolytique, l'action conjuguée d'un air chaud $\left(60^{\circ} \mathrm{C}\right)$ et saturé d'humidité peut hydrolyser jusqu'à $30 \%$ des phytates du blé et des haricots (Fourdin 1984).

- Le $\mathrm{pH}$ : les phytases végétales sont actives à un $\mathrm{pH}$ voisin de 5 ; elles sont très sensibles aux variations de $\mathrm{pH}$, les milieux trop acides ou trop alcalins peuvent les inactiver de façon irréversible. Les phytases végétales des aliments ne peuvent donc être actives que dans l'estomac des animaux, ce qui a été confirmé récemment, chez le porc, à l'aide de canules duodénales (Kemme et Jongbloed 1992). Dans cet essai, il existait une relation linéaire entre l'activité phytasique alimentaire et la dégradation de l'acide phytique mesurée sur le site duodénal. Cette dégradation dépendait également fortement du temps de rétention de l'aliment dans l'estomac.

- La chaleur : pour le blé, le maïs, le triticale et les haricots, l'optimum d'activité est situé autour de $50^{\circ} \mathrm{C}$. L'inactivation par la chaleur prend de l'importance $(50 \%)$ à partir de $70^{\circ} \mathrm{C}$, elle est de $90 \%$ vers $72{ }^{\circ} \mathrm{C}$, pour la phytase du son de blé (Courtois 1947).

Dans la pratique, la question du traitement des aliments et l'échauffement qui en résulte (granulation) peut être décisive pour la conser-
Tableau 5. Répartition comparée de la phytase et des phytates dans le grain de blé (d'après Fourdin 1984, adapté de Reddy et al 1982).

\begin{tabular}{|l|c|c|c|}
\hline Fraction & $\begin{array}{c}\text { Poids relatif } \\
\text { (\% du poids sec } \\
\text { du grain) }\end{array}$ & $\begin{array}{c}\text { Activité phytasique } \\
\text { (en \% de l'activité } \\
\text { totale) }\end{array}$ & $\begin{array}{c}\text { Distribution } \\
\text { des phytates } \\
\text { (en \%) }\end{array}$ \\
\hline Grain entier & 100 & 100 & 100 \\
Endosperme & 82,5 & 34,1 & 2,2 \\
Germe & 1,0 & 2,9 & \} 12,9 \\
Scutellum & 1,5 & 15,3 & \\
Son du blé & 4,5 & 1,9 & 0 \\
- Couches épidermiques & 3,5 & 4,8 & 87,1 \\
- Testa & 7,0 & 39,5 & \\
- Aleurone & & & \\
\hline
\end{tabular}

vation de l'activité phytasique des ingrédients les composant, et en conséquence sur la digestibilité de leur phosphore phytique. Ainsi, pour des aliments composés de matières premières à forte activité phytasique (blé, sons de blé et de seigle, remoulages), le passage en filière (avec conditionnement à la vapeur) n'altère pas cette activité pour une température située entre 47 et $62^{\circ} \mathrm{C}$ (Pointillart et al 1993). Par contre, dans certaines filières, la température peut dépasser $80^{\circ} \mathrm{C}$ et diminuer l'activité (30 à $50 \%$ d'après certaines mesures réalisées au LNSA), voire la supprimer. Ainsi, l'inactivation de la phytase du blé, de l'orge, du remoulage de blé (par la chaleur ou la granulation), réduit considérablement la digestibilité du phosphore végétal chez le Porc (Eeckhout et De Paepe 1992b, Jongbloed et Kemme 1990).

Le froid n'affecterait pas la phytase, mais la congélation entraînerait la formation de cristaux de glace qui, en rompant les membranes, mettrait en présence l'enzyme et son substrat (Fourdin 1984).

D'autres facteurs interfèrent encore avec l'activité de la phytase. Ainsi :

- le calcium (et certains ions métalliques) forme des phytates stables résistant à l'attaque de l'enzyme ;

- la combinaison des phytates avec certaines protéines sous forme de complexes dans les graines les rend moins vulnérables à l'attaque phytasique ;

- la teneur élevée en phytates de certaines farines complètes peut en elle-même inhiber l'action de la phytase. Le taux d'extraction des farines peut donc influer grandement sur la phytase et par la suite sur les effets négatifs des phytates (Pointillart et Guéguen 1992).

La perte de l'intégrité structurale du grain peut modifier son activité phytasique (Reddy et al 1982). Le grain moulu possède une activité phytasique plus importante que le grain entier : le substrat et l'enzyme, séparés dans les conditions physiologiques, sont mis en présence grâce à la destruction partielle des cellules et des membranes. Cela contribue à expliquer la très grande variabilité des données obtenues sur les sons et les remoulages.
Lors de la fabrication des aliments, un chauffage trop fort peut inactiver la phytase naturellement présente dans certaines céréales comme le blé. 


\section{2 / Phytase intestinale}

Les ruminants hydrolysent totalement le phosphore phytique grâce à la flore du rumen. Chez les monogastriques, la situation est variable. Le rat possède une forte activité phytasique, de l'ordre de $30 \mathrm{mUI} / \mathrm{mg}$ de protéines de la muqueuse de l'intestin grêle entier (une UI correspond à une $\mu$ mole de $\mathrm{P}$ hydrolysée/min à $37^{\circ} \mathrm{C}$ et $\mathrm{pH}$ neutre), l'activité pouvant être au moins deux fois plus élevée dans le duodénum. Le poulet aurait une activité phytasique moins négligeable que celle des autres espèces : porc $(0,5$ à $1,5 \mathrm{mUI})$, lapin, cobaye, hamster. Malgré cela, il est paradoxal de constater que la plupart des études in vivo sur la dégradation des phytates et les effets de ceux-ci sur l'indisponiblité des cations ont été réalisées chez les deux espèces présentant la plus forte activité phytasique, le rat et le poulet.

Chez le porc, la phytase du contenu intestinal est également négligeable (confirmé par Jongbloed et al 1992), et il n'y a pas d'adaptation de l'activité phytasique endogène à des apports élevés de phytates. Ainsi, l'utilisation du phosphore et du calcium par le porc diminue au cours du temps lorsque les régimes sont riches en phytates (Pointillart et al 1985). Selon certains auteurs, la phytase serait stimulée par la vitamine $D$; en fait, il semble plutôt que la vitamine $D$, en favorisant l'absorption du calcium au niveau duodénal, rende moins possible la formation de phytates de $\mathrm{Ca}$ insolubles qui sont peu digestibles dans les parties plus distales de l'intestin (Pointillart et al 1989).

Enfin, le phosphore végétal est souvent mieux utilisé par les volailles qu'il ne l'est dans l'espèce porcine (pour des céréales identiques, par exemple : blé, orge, triticale) ; ceci pourrait s'expliquer, au moins en partie, par la présence d'une activité phytasique non négligeable dans leur tube digestif contrairement au porc.

\section{3 / Phytases microbiennes}

\section{a / Historique}

Depuis les premières études de Nelson et al (1971) sur la possibilité d'utiliser des phytases fongiques dans l'alimentation des volailles, des essais avaient été réalisés chez le porc avec des cultures de levures mais sans succès. Ensuite, il a fallu attendre 1990 pour voir publier les premiers travaux sur les possibilités d'améliorer l'utilisation digestive du phosphore chez les porcs et les volailles à partir de 2 phytases produites par culture d'Aspergillus ficuum (Gist-Brocades, Hollande) (Simons et al 1990) et d'Aspergillus niger (Alko, Finlande) (Näsi 1990). Depuis, les travaux se sont multipliés dans la plupart des pays d'Europe (France incluse, essais en cours et Simoes-Nunès 1993) et aux Etats-Unis.

\section{b / Particularités des phytases microbiennes}

"Natuphos", la phytase Gist-Brocades, diffère des phytases végétales par un profil in vitro avec $2 \mathrm{pH}$ optima, l'un à 2,5 et l'autre à 5,5
(Simons et al 1990) ce qui amplifie son champ d'action au niveau de la digestion chez l'animal. Comme la phytase des céréales, les phytases microbiennes sont inactivées par des températures élevées. Ainsi, in vitro, la phytase Gist-Brocades perd $17 \%$ de son activité à $84^{\circ} \mathrm{C}$ et $64 \%$ à $87^{\circ} \mathrm{C}$ (Simons et al 1990), la phytase Alko (Finase) est totalement inactivée au-delà de $60^{\circ} \mathrm{C}$; cette dernière a un profil de pH optimum comparable à la phytase Natuphos (respectivement 2,2 et 5) (Simell et al 1989). Comme pour les phytases de certaines céréales, ces données indiquent que certains procédés de fabrication ou de distribution des aliments peuvent, soit en amorçant l'hydrolyse phytasique (trempage), soit en inhibant l'enzyme (échauffement trop élevé de certaines granulations), considérablement modifier la digestibilité de $\mathrm{P}$ phytique, et qu'en fin l'enzyme s'avère surtout active dans le gastroduodénum, c'est-à-dire à des $\mathrm{pH}$ inférieurs à 6 (Jongbloed et al 1992).

\section{4 / Phytases et digestibilité du phosphore phytique}

\section{a / Phytases végétales des aliments}

Il est certain aujourd'hui que la présence dans les aliments d'une activité phytasique élevée permet une plus grande absorption du phosphore phytique et, partant, végétal. Cela est démontré avec le son de blé (Newton et al 1983), le blé (Pointillart et al 1984), le triticale (Pointillart et al 1987), le son de seigle (Pointillart 1991), le remoulage de blé (Eeckhout et De Paepe 1992b) chez le porc ; avec le triticale chez la poule pondeuse (Sauveur 1984) et avec le blé et l'orge chez le poulet (Sauveur 1983). Notons que chez les volailles, il s'agit de mesure de disponibilité, c'est-à-dire de comparaison, avec une source de $\mathrm{P}$ minéral de référence, de l'efficacité des apports de $\mathrm{P}$ évaluée par la minéralisation osseuse, tandis que, chez le porc, il s'agit de mesures de digestibilité de $\mathrm{P}$, parfois complétées par des mesures de minéralisation osseuse telle que la résistance à la rupture.

Ainsi, en utilisant des mélanges de ces matières premières, on peut se passer complètement de supplémentation en $\mathrm{P}$ minéral tout en obtenant d'excellentes performances zootechniques et une minéralisation osseuse comparable chez le porc (Pointillart et al 1993). En diminuant les apports de $\mathrm{P}$ minéral et en augmentant l'utilisation par l'animal du phosphore végétal, on arrive à réduire de 20 à $30 \%$ les effluents de phosphore (Latimier et Pointillart 1993).

Dans nos essais, chez le porc en croissance en présence d'une activité phytasique élevée (jusqu'à $1200 \mathrm{U} / \mathrm{kg}$ d'aliment), des gains de digestibilité du phosphore végétal d'environ $20 \%$ ont été obtenus, chiffre comparable à celui observé en Belgique (Eeckhout et De Paepe $1992 \mathrm{~b}$ ) avec un régime incorporant $10 \%$ de remoulage de blé (soit, dans cet essai, environ $500 \mathrm{U}$ de phytase $/ \mathrm{kg}$ ). Chez les volailles (Sauveur 1989), la disponibilité du phosphore 
végétal avoisine $50 \%$ pour les céréales à forte activité phytasique contre $20 \%$ environ pour les autres. Cela devrait donc correspondre à un gain de digestibilité légèrement supérieur à celui observé chez les porcins (en admettant une relation linéaire entre disponibilité et digestibilité).

\section{b / Phytases microbiennes ajoutées}

Aujourd'hui, il est bien démontré, tant chez les volailles (Sauveur 1993) que chez le porc, que l'addition de phytase microbienne améliore notablement la rétention du phosphore végétal. Par exemple, dans les essais réalisés en Hollande chez le poulet de chair, l'effet sur la rétention de $\mathrm{P}$ végétal est presque linéaire entre 250 et 1500 U d'enzy$\mathrm{me} / \mathrm{kg}$ (phytase Gist-Brocades) tandis que chez le porc (35-70 kg), l'addition de $1000 \mathrm{U} / \mathrm{kg}$ d'aliment augmente la digestibilité du $P$ végétal de 22 à 26 points suivant la nature des régimes (Simons et al 1990). Dans ces essais, les rejets de $\mathrm{P}$ diminuaient de $50 \%$ chez les poulets et de $35 \%$ chez les porcs. En Suisse (Kessler et Egli 1992), en réduisant de $32 \%$ les apports de $\mathrm{P}$ chez les porcs en croissancefinition par l'utilisation de $500 \mathrm{U} / \mathrm{kg}$ de phytase (Finase, Alko), une réduction de $50 \%$ de l'excrétion totale de $\mathrm{P}$ est observée. En Finlande, la même dose (Finase) permet une amélioration de 24 points de la digestibilité du $\mathrm{P}$ végétal chez le porc (Näsi 1990). En Allemagne, des gains de digestibilité de 26 $(500 \mathrm{U})$ à 35 points $(1000 \mathrm{U}$, phytase GistBrocades) sont obtenus chez des jeunes porcs, équivalant à une économie de $0,2 \%$ de $\mathrm{P}$ minéral et une réduction de l'excrétion fécale de $\mathrm{P}$ de 50\% (Pallauf et al 1992). Aux EtatsUnis, Cromwell (1991) constate une amélioration de la résistance à la rupture des os chez des porcs en finition ne recevant pas de $P$ minéral, en incorporant $500 \mathrm{U} / \mathrm{kg}$ (Finase), l'effet étant accru avec $1000 \mathrm{U}$. Dans cet essai, Cromwell estime de 35 à $50 \%$ (suivant qu'il se réfère aux normes d'apports de $\mathrm{P}$ du NRC ou à celles réellement pratiquées aux Etats Unis), la réduction possible de l'excrétion de $\mathrm{P}$. Il évalue à $2,3 \mathrm{~g}$ de $\mathrm{P}$ digestible l'effet de $1000 \mathrm{U} / \mathrm{kg}$ ce qui, d'après lui, permettrait de pratiquement supprimer la complémentation en $\mathrm{P}$ minéral avec les régimes mais-soja. Des résultats obtenus en Belgique (Eeckhout et De Paepe 1992b), il est déduit que $500 \mathrm{U} / \mathrm{kg}$ de "Natuphos" permettent d'économiser (par kg d'aliment) $0,8 \mathrm{~g}$ de P digestible, soit environ $6,3 \mathrm{~g}$ de phosphate bicalcique. Les conclusions hollandaises (Beers 1992) évaluent à $0,7-0,8 \mathrm{~g} / \mathrm{kg}$ d'aliment les économies de $\mathrm{P}$ digestible réalisées avec 500 UI (Natuphos), celles réalisables avec des régimes porcs maïs-soja étant plus spectaculaires que celles avec les régimes hollandais courants, riches en produits de substitution des céréales (PSC). Ainsi, avec $300 \mathrm{U} / \mathrm{kg}$, le gain de $\mathrm{P}$ digestible est déjà de $0,7 \mathrm{~g} / \mathrm{kg}$ avec un régime mails-soja contre $0,4 \mathrm{~g}$ pour un régime à base de PSC ; à partir de $1000 \mathrm{U} / \mathrm{kg}$, la digestibilité de $\mathrm{P}$ végétal avoisine $60 \%$ pour les 2 types de régimes.

\section{c / Phytase végétale et phytase microbienne}

L'efficacité de la phytase du blé (remoulage) et de celle d'Aspergillus (Gist Brocades), à raison de $500 \mathrm{U} / \mathrm{kg}$ d'aliment, ont été comparées chez des porcelets et des porcs en croissance (Eeckhout et De Paepe 1992a, b et c). L'écart entre les 2 phytases atteint presque $10 \%$ (il est significatif seulement chez les porcelets) pour ce qui concerne les digestibilités du phosphore. Selon Eeckhout et De Paepe, la plus grande efficacité de la phytase microbienne viendrait de son profil de $\mathrm{pH}$ plus étendu que celle de la phytase du blé. Ils observent également que l'effet de la phytase microbienne est plus prononcé lorsque le régime ne contient pas de phytase propre, en particulier quand il ne contient pas de blé (Eeckhout et De Paepe 1992a). Cela est également constaté chez les volailles (Six 1992). Par contre, chez le porc, il pourrait y avoir additivité des 2 types de phytase (Eeckhout et De Paepe 1992b), ce qui n'est pas forcément acquis chez les volailles (Six 1992). Dans tous ces essais, l'amélioration de l'utilisation du phosphore s'accompagnait la plupart du temps d'une amélioration de l'absorption du calcium (Simons et al 1990, Pallauf et al 1992), voire, chez les volailles, d'une amélioration des performances (Simons et al 1990, Dudley-Cash 1991).

\section{d / En conclusion}

Les phytases, végétales ou microbiennes, influencent notablement la digestibilité du phosphore végétal, l'amélioration étant de l'ordre de 20 points pour les phytases des céréales (ou de leurs issues) et de 25-30 points ou plus pour les phytases microbiennes. Cela engendre des économies importantes dans l'apport de $\mathrm{P}$ minéral $(0,7$ à $1 \mathrm{~g} / \mathrm{kg}$ suivant les espèces et les régimes, soit entre 6 et $7 \mathrm{~g}$ de phosphate bicalcique/kg), voire, avec certains régimes (ceux composés de céréales et d'issues à forte activité phytasique) ou certaines doses de phytase microbienne ( $\geq 1000 \mathrm{U} / \mathrm{kg}$ ), sa suppression totale tout au moins chez le porc. Les réductions possibles des effluents de $\mathrm{P}$ qui en découlent varient de 20 à $50 \%$ suivant les travaux. Ce dernier point présente un intérêt évident dans les zones de fortes concentrations d'élevages de porcs et de volailles telles qu'en Bretagne ou aux Pays-Bas. Les précautions consistent essentiellement à surveiller l'intégrité de l'activité phytasique en évitant le surchauffage (au-delà de $70 / 75^{\circ} \mathrm{C}$ ) au cours de la fabrication des aliments. De ce point de vue la phytase microbienne pourrait présenter une certaine souplesse d'emploi, puisque l'on peut en ajouter suffisamment compte tenu d'un certain pourcentage d'inactivation dans les filières (environ $30 \%$ selon nos estimations). Toutefois, le problème ne semble pas complètement résolu puisque les Hollandais préconisent l'utilisation de soupes chez les porcs et évitent la granulation. Enfin, il est évident, notamment à partir des données fournies sur les phytases végétales, qu'il ne suffit pas de connaitre la teneur en phosphore phytique des ingrédients pour en déduire la digestibilité de

\section{L'addition de phytase d'origine microbienne permet d'améliorer l'utilisation du P contenu dans les aliments et donc de réduire la supplémentation en P minéral.}


leur $\mathrm{P}$ végétal, puisqu'à teneur égale, elle peut être très différente suivant qu'ils renferment ou non une activité phytasique importante une fois l'aliment fabriqué.

\section{3 / Disponibilité et digestibilité du phosphore végétal}

Les mesures de digestibilité correspondent, chez le porc, à des mesures des excrétions fécales de phosphore déduites du phosphore ingéré : digestibilité = (ingéré-fécal) \% ingéré. Ces mesures n'étant pas réalisables chez les volailles (fèces et urines mélangées), on a recours à des mesures, soit de rétention (ingéré-excrété total, urine+fèces), soit à des mesures de disponibilité, en évaluant la minéralisation osseuse (contenu en cendres ou résistance à la rupture d'un os type) avec une source végétale donnée de phosphore comparée à une source de phosphate minéral à qui est attribuée la valeur 100 . Ainsi, quand on dit que la disponibilité de $\mathrm{P}$ végétal du blé est de $49 \%$, cela signifie que son efficacité (ou son utilisation par l'animal) équivaut à $49 \%$ de celle d'un bon phosphore minéral (phosphate bicalcique ou phosphate monosodique ou un autre). La disponibilité ne correspond donc pas à la digestibilité, un très bon phosphate n'étant pas absorbé à $100 \%$ (environ $80 \%$ ). Cette méthode est également utilisée aux Etats-Unis pour les porcs, le plus souvent à partir de tests de résistance à la rupture de certains os (et par comparaison au phosphate monosodique) (cf. Cromwell 1992, pour plus de détails). On peut donc aussi utiliser le terme de biodisponibilité puisqu'il s'agit d'une mesure réalisée sur l'animal. Dans l'ensemble, les mesures de disponibilité (ou de biodisponibilité) chez les volailles sont plus fiables que celles réalisées chez le porc, du fait des possibilités de disposer de beaucoup plus grands effectifs d'animaux.
Ainsi, les mesures de digestibilité réalisées chez les porcs portent souvent sur moins d'une dizaine d'animaux par matière première (Jongbloed et al 1991), et elles comportent également une part d'extrapolation importante. Par contre, si les valeurs prises en compte pour la formulation chez les volailles sont basées réellement sur des mesures de disponibilité, le plus souvent encore chez les porcs, en France tout au moins, les valeurs de disponibilité données par les logiciels reposent sur des extrapolations à partir du contenu en $\mathrm{P}$ non phytique des matières premières ou, au mieux, sur des estimations à partir des diverses valeurs de disponibilité vraie (mesures osseuses comparatives) ou de digestibilité publiées dans la littérature internationale. Les tables de recommandations INRA pour les porcs, formulées en $P$ total, tiennent compte, avec une bonne marge d'erreur, de la digestibilité moyenne de $\mathrm{P}$ des matières premières végétales (environ $30 \%$ contre plus de $70 \%$ pour le $\mathrm{P}$ minéral). Cela signifie qu'elles sont perfectibles : lorsqu'on connaîtra suffisamment bien la digestibilité (ou la biodisponibilité) de toutes les matières premières végétales (y compris dans diverses situations alimentaires : avec ou sans phytase), on pourra alors formuler en $\mathrm{P}$ digestible (ou en $\mathrm{P}$ disponible) comme pour les volailles, ce qui sans doute conduira à des économies de phosphore.

Le tableau 6 illustre ce qui vient d'être dit. Pour le blé ou le maïs, il y a un assez bon accord entre les diverses évaluations de la portion du phosphore végétal utilisable par l'animal. Toutefois, pour le blé, le $\mathrm{P}$ utilisable correspond plutôt à la valeur la plus élevée du phosphore végétal non phytique de la céréale tandis que pour le maïs, il correspond plutôt à la valeur la plus faible.

Cela traduit le souci des uns et des autres de prendre une marge de sécurité nécessairement plus grande pour le maïs que pour le blé

Tableau 6. Disponibilité du phosphore végétal en fonction des espèces et des modes d'évaluation.

\begin{tabular}{|c|c|c|c|c|c|c|}
\hline & $\begin{array}{c}\mathrm{A} \\
\mathrm{P} \text { total } \\
\mathrm{g} / \mathrm{kg}\end{array}$ & $\begin{array}{c}\mathrm{B} \\
\mathrm{P} \text { végétal } \\
\text { non phytique } \\
\% \text { P total }\end{array}$ & $\begin{array}{c}\text { C } \\
\text { "P disp." } \\
\text { PORFAL } \\
\%\end{array}$ & $\begin{array}{c}\text { Porcs } \\
\text { D } \\
\text { P dig. } \\
\text { Jongbloed } \\
\text { et al \% }\end{array}$ & $\begin{array}{c}\mathrm{E} \\
\text { P biod. } \\
\text { Cromwell } \\
\%\end{array}$ & $\begin{array}{c}\text { Volailles } \\
\text { F } \\
\text { P disp. } \\
\text { Sauveur } \\
\%\end{array}$ \\
\hline Blé & 3,3 & $23-40$ & 45 & 47 & 49 & 55 \\
\hline Orge & 3,5 & $34-49$ & 29 & 39 & 30 & 49 \\
\hline Maïs & 2,7 & $15-34$ & 15 & 17 & 14 & 18 \\
\hline Tourteau de soja & 6,9 & $39-49$ & 38 & 38 & $23-31$ & 15 \\
\hline Tourteau de colza & 10,6 & $27-40$ & 27 & - & - & 22 \\
\hline Pois & 4,5 & $50-60$ & 42 & 45 & - & 35 \\
\hline Remoulage de blé & 6,7 & $15-34$ & 30 & 28 & 41 & - \\
\hline Sons de blé & 12 & $10-30$ & 30 & - & 29 & 55 \\
\hline
\end{tabular}

A et $\mathrm{C}$ : valeurs données par le logiciel ITP-PORFAL, $\mathrm{B}:$ valeurs calculées d'après le tableau $3, \mathrm{C}:$ phosphore disponible en \% du phosphore total selon le logiciel PORFAL-ITP, D : mesures de digestibilité, sur 5 à 7 animaux suivant les matières premières (Jongbloed et al 1991), E : valeurs de biodisponibilité (= bioavailability) comparative par rapport au phosphate monosodique à qui est attribuée la valeur 100, test de résistance à la rupture, mesures sur 2 à 7 tests (Cromwell 1992), $\mathrm{F}$ : mesures de disponibilité réellement effectuées par des tests osseux (= biodisponibilité comme pour Cromwell) en référence le plus souvent au phosphate monocalcique (=100\%) (Sauveur 1989). 


\section{Exemple de calculs d'économie de phosphore possible avec la phytase.}

Besoin moyen d'un porc en finition (de 50 a $100 \mathrm{~kg}$ ) : estimé à $2,5 \mathrm{~g}$ de P digestible par $\mathrm{kg}$ d'aliment $(5 \mathrm{~g}$ de $P$ total digestible à $50 \%$ ). Régime céréales + tourteaux : apport de $P$ végétal estimé à $4 \mathrm{~g} / \mathrm{kg}$ daliment

\begin{abstract}
Digestibilité de P végétal
$P$ végétal digestible

A ajouter sous forme de $P$ digestible minéral

Ce qui équivaut à un apport de $P$ minéral

(en affectant à celui-ci une digestibilité moyenne de $80 \%$ )

(notamment, parce que ce dernier possède une activité phytasique importante). On remarque quelques variations interspécifiques : le phosphore du tourteau de soja semble moins assimilable chez les volailles que chez les porcs, de même pour celui du pois, par contre celui des sons de blé et des orges est plus utilisable en moyenne chez les volailles que chez les porcs. Pour certaines matières premières comme l'orge et les remoulages (mais aussi beaucoup d'autres), les différentes valeurs de l'estimation de $\mathrm{P}$ utilisable par l'animal concordent mal et des mesures complémentaires semblent requises.
\end{abstract}

$$
1,1 / 0,8=1,4 \mathrm{~g} / \mathrm{kg} \quad 0,5 / 0,8=0,6 \mathrm{~g} / \mathrm{kg}
$$

Soit $57 \%$ de phosphate en moins à incorporer dans le régime avec phytase $[(1,4-0,6) / 1,6 \times 100]$
$P$ total du régime, $\mathrm{g} / \mathrm{kg}$
$4+1,4=5,4$
$4+0,6=4,6$

avec $500 / 1000 \mathrm{U} / \mathrm{kg}$

$$
>50 \%
$$

$0,5 \times 4=2 \mathrm{~g} / \mathrm{kg}$

$2,5-2=0,5 \mathrm{~g} / \mathrm{kg}$
Soit une réduction de l'apport total de $\mathrm{P}$ de $15 \%[(5,4-4,6) / 5,4 \times 100]$ avec le régime "phytase".
Excrétion fécale de $P$
$5,4-2,5=2,9$
$4,6-2,5=2,1$

Soit une réduction de l'excrétion fécale de $P$ de près de $28 \%(2,9-2,1 / 2,9 \times 100]$

\section{Conclusions}

La totalité du phosphore phytique, abondant dans les ingrédients composant les aliments pour porcs et volailles, n'est pas inutilisable par l'animal. Cela dépend de la nature du produit considéré et en particulier de la persistance ou non, dans l'aliment après sa fabrication, d'une forte activité phytasique ( $\geq 500 \mathrm{U} / \mathrm{kg}$ ?). Ainsi peut-on, chez les porcs, en utilisant du blé et des sous-produits du blé (voire du seigle ou du son de seigle) se passer de l'addition de $\mathrm{P}$ minéral. Chez les volailles, exceptées les poules pondeuses (par exemple en utilisant le triticale), cela semble moins possible. Par contre, chez ces dernières comme chez les porcs, l'incorporation de phytases microbiennes (entre 500 et $1000 \mathrm{UI} / \mathrm{kg}$ ) peut permettre, en valorisant le phosphore végétal, c'est-à-dire en augmentant considérablement la digestibilité des phytates (comme le font, peut-être à moindre degré, les phytases des céréales), de réduire considérablement et parfois totalement (porcs) la nécessité d'ajouter des phosphates minéraux. A condition de vérifier que l'activité phytasique incorporée subsiste au moment de la consommation de l'aliment par l'animal, ce qui vaut également pour les phytases végétales. L'avantage des phytases microbiennes porte surtout sur un "dosage" plus aisé lors de son incorporation : on commence à connaître assez bien les digestibilités correspondant à certaines doses tandis que pour les phytases des céréales et des issues une grande incertitude subsiste quant à leurs activités enzymatiques moyennes (et les digestibilités correspondantes), faute d'études systématiques. Il est néanmoins certain que la phytase du blé agit sur d'autres phytates que ceux du blé (Scheuermann et al 1988). L'avantage des phytases naturelles est qu'elles ne coûtent rien puisqu'elles sont déjà présentes dans le blé, le triticale, le seigle et leurs sous-produits riches en enveloppes (sons et remoulages) et qu'il suffit d'en incorporer des proportions significatives (10 à $20 \%$ d'issues par exemple) dans les aliments, pour améliorer la digestibilité de leur $\mathrm{P}$ végétal. Ce procédé peut s'avérer intéressant en France où les céréales, et notamment le blé, sont produites en abondance ; la situation semble un peu différente aux Pays-Bas avec la forte utilisation de PSC et la cherté du blé. L'un comme l'autre de ces procédés, phytases microbiennes ou céréalières, permet de réduire, entre 20 et $50 \%$ (cf. encart), les effluents de phosphore, ce qui n'est pas négligeable et, pour certaines zones d'élevage, contribuerait à diminuer les risques d'eutrophisation des eaux. La généralisation de ces procédés devrait être précédée d'études plus complètes sur les facteurs physico-chimiques et biochimiques interférant avec l'activité phytasique (teneur en calcium des rations, influence des modes de fabrication et de distribution, conservation, etc.). 


\section{Références bibliographiques}

Beers S., 1992. Relation between the dose of microbial phytase and phosphorus digestibility in diets for pigs. Rapport IVVO-DLO n²28, $40 \mathrm{pp}$.(Lelystad, Nederland).

Bos K.D., 1986. Chemical background of phosphorus compounds and phytase in livestock feed. Rapport CIVO-TNO n ${ }^{\circ}$ A $85560,35 \mathrm{pp}$.

Courtois J., 1947. Recherches sur la phytase III. Essais de séparation de l'activité glycérophosphatasique et de l'activité phytasique du son de blé. Bioch. Biophys. Acta, 1, 270-277.

Cromwell G.L., 1991. Phytase appears to reduce phosphorus in feed, manure. Feedstuffs, 63, 14-16.

Cromwell G.L., 1992. The biological availability of phosphorus in feedstuffs for pigs. Pigs News Information, 13, 75N-78N.

Dudley-Cash W.A., 1991. Microbial phytase effects phytate phosphorus digestibility, excretion. Feedstuffs, 63, 12.

Eeckhout W., De Paepe M., 1992a. Influence d'une phytase microbienne sur la digestibilité apparente du phosphore d'aliments pour porcelets. Revue de l'Agriculture, 45, 183-193.

Eeckhout W., De Paepe M., 1992b. Phytase de blé, phytase microbienne et digestibilité apparente du phosphore d'un aliment simple pour porcelets. Revue de l'Agriculture, 45, 195-207.

Eeckhout W., De Paepe M., 1992c. Comparaison de l'effet de 500 unités de phytase de blé et d'une phytase microbienne sur la digestibilité apparente du phosphore d'un aliment pour pores à l'engrais. Revue de l'Agriculture, 45, 209-216.

Erdman J.W., 1979. Oilseed phytates : Nutritional implications. J. Am. Oil. Chem. Soc., 56, 736-741.

Fourdin A., 1984. Valorisation par la phytase du phosphore phytique des grains. I. Introduction bibliographique sur la phytase. DEA Sciences Alimentaires 1984, Paris XI-ENSIA Massy, 18 pp (LNSA).

Guéguen L., Besançon P., Rérat A., 1968. Utilisation digestive, cinétique de l'absorption et efficacité de la rétention du phosphore phytique chez le porc. Ann. Biol. Anim. Bioch. Biophys., 8, 273-280.

INRA, 1989. L'alimentation des animaux monogastriques, porc, lapin, volailles. Ouvrage collectif. $2^{\text {ème }}$ édition INRA, Paris, $282 \mathrm{pp}$.

Jongbloed A.W., Kemme P.A., 1990. Effect of pelleting mixed feeds on phytase activity and the apparent absorbability of phosphorus and calcium in pigs. Anim. Feed Sci. Technol., 28, 233-242.

Jongbloed A.W., Evert S.H., Kemme P.A., 1991. Phosphorus availability and requirements in pigs. Rec. Adv. Anim. Nutr., 4, 65-80.

Jongbloed A.W., Mroz Z., Kemme P.A., 1992. The effect of supplementary Aspergillus Niger phytase in diets for pigs on concentration and apparent digestibility of dry matter, total phosphorus, and phytic acid in different sections of the alimentary tract. J. Anim. Sci., 70, 1159-1168.
Kemme P.A., Jongbloed A.W., 1992. Degradation of phytic acid in the stomach of the pig as influenced by phytase of plant origin. Rapport IVVO-DLO n 236 27 pp. (Lelystad, Nederland).

Kessler J., Egli K., 1992. Phosphor sparen dank Phytase : Erste Ergebnisse beim Mastschwein. Landwirtschaft. Schweiz. Band, 5, 5-9.

Latimier P., Pointillart A., 1993. Influence de l'apport de phosphore $(0,4-0,5-0,6)$ sur les performances, les rejets de phosphore et la minéralisation osseuse chez le porc charcutier. Jour. Rech. Porc., 25, 277-286.

Maupetit P., Gatel F., 1992. Phosphore phytique dans les matières premières : variabilité de la teneur en phosphore phytique du blé, de l'orge et du pois. Colloque annuel CRITT-VALICENTRE, Orléans, Octobre 1992, 15-23.

Näsi M., 1990. Microbial phytase supplementation for improving availability of plant phosphorus in the diet of the growing pigs. J. Agric. Sci. (Finland), 162, 435-443.

Nelson T.S. Shieh R.R., Wodzinski R.J., Ware J.H., 1971. Effect of supplemental phytase on the utilization of phytate phosphorus by chicks. J. Nutr., 101, 1289-1293.

Newton G.L., Hale O.M., Plank C.O., 1983. Effect of wheat bran in practical diets on mineral absorption by pigs at two ages. Can. J. Anim. Sci., 63, 399-408.

Oberleas D., Muhrer M.E., O'Dell B.L., 1962. Effects of phytic acid on zinc availability and parakeratosis in swine. J. Anim. Sci., 21, 57-61.

Pallauf Von J., Hohler D., Rimbach G., Neusser H., 1992. Einfluss einer Zulage an mikrobieller Phytase $\mathrm{zu}$ einer Mais-Soja-Diät auf die scheinbare Absorption von Phosphor und Calcium beim Ferkel. J. Anim. Physiol. Anim. Nutr., 67, 30-40.

Pointillart A., 1991. Enhancement of phosphorus utilization in growing pigs fed phytate-rich diets by using rye bran. J. Anim. Sci., 69, 1109-1115.

Pointillart A., Guéguen L., 1992. Influence des fibres alimentaires sur la biodisponibilité des minéraux. Cahiers ENSBANA, 18, 157-182.

Pointillart A., Fontaine N., Thomasset M., 1984 Phytate phosphorus utilization and intestinal phosphatases in pigs fed low phosphorus: wheat or corn diets. Nutr. Rep. Int., 29, 473-483.

Pointillart A., Fontaine N., Thomasset M., Jay ME. 1985. Phosphorus utilization, intestinal phosphatases and hormonal control of calcium metabolism in pigs fed phytic phosphorus : soyabean or rapeseed diets. Nutr. Rep. Int., 32, 155-167.

Pointillart A., Fourdin A., Fontaine N., 1987. Importance of cereal phytase activity for phytate phosphorus utilization by growing pigs fed diets containing triticale or corn. J. Nutr., 117, 907-913.

Pointillart A., Colin C., Cayron B., Camus P., Fourdin A., 1989. Apport vitaminique D et absorption du phosphore phytique chez le porc. Jour. Rech. Porc., 21, 39-44. 
Pointillart A., Colin C., Lacroix C., Radisson J., 1993. Réduction chez le porc en croissance de la supplémentation en phosphore minéral par l'utilisation de céréales à activité phytasique élevée. Jour. Rech. Porc., 25, 233-238.

Reddy N.R., Sathe S.K., Salunkhe D.K., 1982. Phytates in legumes and cereals. Adv. Food. Res., 28, $1-92$.

Sauveur B., 1983. Bio-availability to poultry of plant-origin phosphorus. Methodological criticisms and results. Proc. 4th. Europ. Poultry Nutrition, Tours, 103-113.

Sauveur B., 1984. High availability of triticale phosphorus for laying hens. Nutr. Rep. Int., 29, 911-919.

Sauveur B., 1989. Phosphore phytique et phytases dans l'alimentation des volailles. INRA Prod. Anim., 2, 343-351.

Sauveur B., 1993. Les phytases fongiques dans l'alimentation des volailles. INRA Prod. Anim., 6, 265-267.

Scheuermann Von S.E., Lantzsch H.J., Menke K.H., 1988. In vitro und in vivo Untersuchungen zur Hydrolyse von Phytat. II. Aktivität pflanzlicher Phytase. J. Anim. Physiol. Anim. Nutr., 60, 64-75.
Simell M., Turunen M.P., Ronen J., Vaara T., 1989. Feed and food applications of phytase. 3rd meeting on industrial applications of enzymes. Barcelona, December 1989, 15 pp.

Simoes-Nunès C., 1993. Effets zootechniques et biochimiques de l'addition de phytase au régime alimentaire du porc. Jour. Rech. Porc., 25, 229-232.

Simons P.C.M., Versteegh H.A.J., Jongbloed A.W., Kemme P.A., Slump P., Bos K.D., Wolters M.G.E., 1990. Improvement of phosphorus availability by microbial phytase in broiler and pigs. Brit. J. Nutr., 64, 525-540.

Six L., 1992. Intérêt des phytases microbiennes dans l'alimentation du poulet de chair. Mémoire maîtrise Sci. Tech. Prod. Anim., 34 pp. (INRA-Nouzilly, Univ F. Rabelais, Tours).

\section{Remerciements}

La rédaction de cette revue a été facilitée par les résultats d'analyse (phytase et phytates) obtenus au Laboratoire de Nutrition et Sécurité Alimentaire (Jouy-en-Josas) par Colette Colin

\section{Summary}

\section{Phytates and phytases: their relevance in the} feeding of pigs and poultry.

Plant, mainly phytic (2/3-3/4), phosphorus assumed to be poorly available usually provides $2 / 3$ to $3 / 4$ of the total $P$ ingested by monogastric animals. Its availability may be improved either by adding microbial phytase to diets or by using phytase-rich cereal diets. Some cereals as wheat or rye or its by-products exhibit high phytase activity. Availability of plant $P$ may vary from less than $20 \%$ for diets devoided of phytase activity to more than $60 \%$ for diets containing near 1000 microbial phytase units/kg. This may optimize the plant $\mathbf{P}$ utilization by pigs and poultry, saving inorganic $P$ addition (up to $100 \%$ ), thus markedly reducing (up to $50 \%$ ) excreted $P$ in manure. The main restriction to the extensive use of phytases, whatever their origin : phytaserich cereals or added microbial phytases, depends on the temperature reached during feed processing. Some heating associated to pelleting process $\left(70-80^{\circ} \mathrm{C}\right)$ partially $(30$ to $50 \%)$ inactivate the enzymatic activity, which is generally completely inactivated over $80^{\circ} \mathrm{C}$. There is also a lack of screening of the relationships between cereal phytase levels and the corresponding phytic $P$ availabilities.

POINTILLART A., 1994. Phytates, phytases : leur importance dans l'alimentation des monogastriques. INRA Prod. Anim., 7 (1), 29-39. 